\title{
Changes in smokers' behavior following the implementation of a smoke-free apartment-building legislation
}

\author{
Ji-eun Hwang ${ }^{1}$, Sung-il Cho ${ }^{1,2}$, Eonjoo Park ${ }^{2}$
}

\begin{abstract}
INTRODUCtION It has been noted in South Korea since September 2016 that at least half of the households residing in an apartment building were in favor of designating common areas such as the hallway, stairway, elevator and underground parking-lot as non-smoking areas. The purpose of this study is to examine changes in smokers' behavior following the implementation of this smoke-free apartment building legislation.

METHODS A cross-sectional mobile survey was conducted among a convenience sample of residents of four apartment buildings in three regions of South Korea. The survey was distributed via notices posted on each apartment's bulletin board for about one month. The apartment buildings' adult residents were able to access the questionnaire using the Quick Response (QR) code provided on the notice. RESULTS A total of 378 residents, 38 of whom were smokers, participated in the mobile survey. Following the implementation of the smoke-free apartment regulations, smoking was reduced in public areas (e.g. hallways, stairways, and underground parking-lots) while smoking activity in other areas increased. Furthermore, $39.5 \%(n=15)$ of current smokers had attempted to quit, and $23.7 \%$ $(n=9)$ who smoked outside $(n=6)$ and inside the buildings $(n=3)$ had changed their habits.

CONCLUSIONS The designation of non-smoking areas in apartment buildings may have had a positive effect on smokers' habits in the short-term. In the future, efforts to support smoking cessation will be necessary for the creation of smoke-free community spaces, i.e. smoke-free areas in apartment buildings.
\end{abstract}

\author{
AFFILIATION \\ 1 Institute of Health and \\ Environment, Seoul National \\ University, Seoul, Republic \\ of Korea \\ 2 Department of Public \\ Health Sciences, Graduate \\ School of Public Health, Seoul \\ National University, Seoul, \\ Republic of Korea \\ CORRESPONDENCE TO \\ Sung-il Cho. Department \\ of Public Health Sciences, \\ Graduate School of Public \\ Health, Seoul National \\ University, 1 Gwanak-ro, \\ Gwanak-gu, Seoul 08826, \\ Republic of Korea. E-mail: \\ persontime@hotmail.com \\ KEYWORDS \\ smoke-free, smoking, tobacco, \\ multi-family housing, \\ apartment
}

Received: 27 August 2019 Revised: 11 November 2019 Accepted: 14 November 2019

\section{INTRODUCTION}

Article 8 of the Framework Convention on Tobacco Control (FCTC) urges all relevant parties to implement effective regulatory and administrative measures aimed at prohibiting smoking in indoor public places, for the purpose of protecting current and future generations from the health, social, environmental and economic damage caused by exposure to tobacco smoke $^{1}$. The average rate of implementation of FCTC Article 8 worldwide is $88 \%$, which is higher than that of all other similar provisions ${ }^{2}$. However, the rate of implementation of a complete ban varies among locations in different countries ${ }^{2}$. Areas that have been designated as completely smoke-free spaces have high implementation rates (95\% for airplanes, $87 \%$ for public transportation, and $87 \%$ for educational facilities); by contrast, the implementation rate for private vehicles is low (16\%). The extent of nonsmoking areas in multi-unit housing complexes remains unknown.

Smoking in the public areas of apartment buildings, such as hallways, stairways, elevators, and underground parking-lots, can be a significant source of conflict among neighbours. In South Korea, since more people live in apartments than in single-family homes, smoking in the public areas of apartment complexes 
is a major contributor to secondhand smoking. The damage caused by secondhand smoke is serious, and targeted countermeasures are required to prevent it. To that end, the government of South Korea revised the National Health Promotion Act to implement a measure that allows residents of apartment buildings to self-designate non-smoking areas. According to the revised law, if more than half of the households in an apartment building are in favor of designating all or part of the four key areas-hallways, stairways, elevators and underground parking-lots-as nonsmoking spaces, the apartment building's director can submit a request to the mayor, or the county or district head office of the apartment complex, regarding the designation of non-smoking areas; the mayor or county or district head office is then required to designate them as such. As in other nonsmoking areas, a fine is imposed for smoking in these spaces, with a penalty of $50000 \mathrm{KRW}$ (South Korean Won), about 41 US $\$$.

The spread of non-smoking areas in apartment buildings has been slow. As of June 2018, only 631 apartment buildings nationwide had adopted the smoke-free regulations, despite the measure having been introduced on 3 September 2016. This is equivalent to $3 \%$ of South Korea's apartment buildings. Owing to the minimal enforcement of the regulations, domestic and foreign studies on this subject have hitherto been scarce ${ }^{3}$. In particular, few studies have investigated the effects of designated non-smoking areas on smokers' smoking habits. Therefore, the purpose of this study is to ascertain whether the designation of non-smoking areas in South Korea has affected smokers' behavior, such as the extent to which they smoke, where they choose to smoke, and smoking cessation attempts.

\section{METHODS}

A cross-sectional mobile survey was conducted, targeting individuals aged over 19 years who had been resident in selected apartment buildings for longer than six months. The apartments were selected according to region and size from among apartment buildings with designated non-smoking hallways, stairways, elevators, and underground parking-lots. Finally, four apartment buildings in three areas (Seoul Metropolitan City, Daejeon Metropolitan City, and Gyeonggi-do Province) were targeted, with notices regarding the mobile survey being posted on bulletin boards by the apartment management offices. The mobile survey application used in this study is a selfenrollment program accessed by a Quick Response (QR) code included in the accompanying guide, which allows individuals to participate in surveys after providing consent. The survey notices were posted for various periods of time, differing among the apartment buildings, and the survey period was from 20 October 2018 to 25 November 2018. Individuals participating in the survey received e-vouchers for convenience stores worth $5000 \mathrm{KRW}$ (about 4.1 US\$), as compensation for their time. This study was approved by the Institutional Review Board of the Seoul National University (No.1809/003-007).

\section{Measures}

The participants were asked about their smoking habits. They indicated the areas in which they smoked, both before and after the designation of smoke-free spaces in their apartment buildings, from a list that included hallways, stairways, elevators, underground parking-lots, apartments, and other areas. Participants were also asked whether their smoking habits had changed after the designation of non-smoking areas in their apartment building, and whether they had actually attempted to quit smoking.

\section{Analysis}

This study used R software (ver. 3.5.3; R Development Core Team, Vienna, Austria) to generate descriptive statistics on the participants' demographic characteristics as frequencies and percentages. Smoking-habit data, analyzed as frequencies, were plotted using the ggplot package, and the McNemar test was used to compare the data collected before and after the intervention.

\section{RESULTS}

A total of 378 people participated in the mobile survey, including 191 men (50.5\%) and 187 women (49.5\%). Smoking was strictly prohibited in most of their residences $(88.4 \% ; n=334)$, but $9.0 \%(n=34)$ were occasionally allowed to smoke while $2.6 \%(n=10)$ were permitted to smoke freely. In total, 38 respondents (10.1\%) were current smokers, and 340 (89.9\%) were currently non-smokers; $14 \%(n=49)$ of the nonsmokers shared their households with smokers.

Of the 38 smokers, 15 (39.5\%) indicated that they 
Figure 1. Smoking areas before and after implementation of smoke-free apartment building legislation

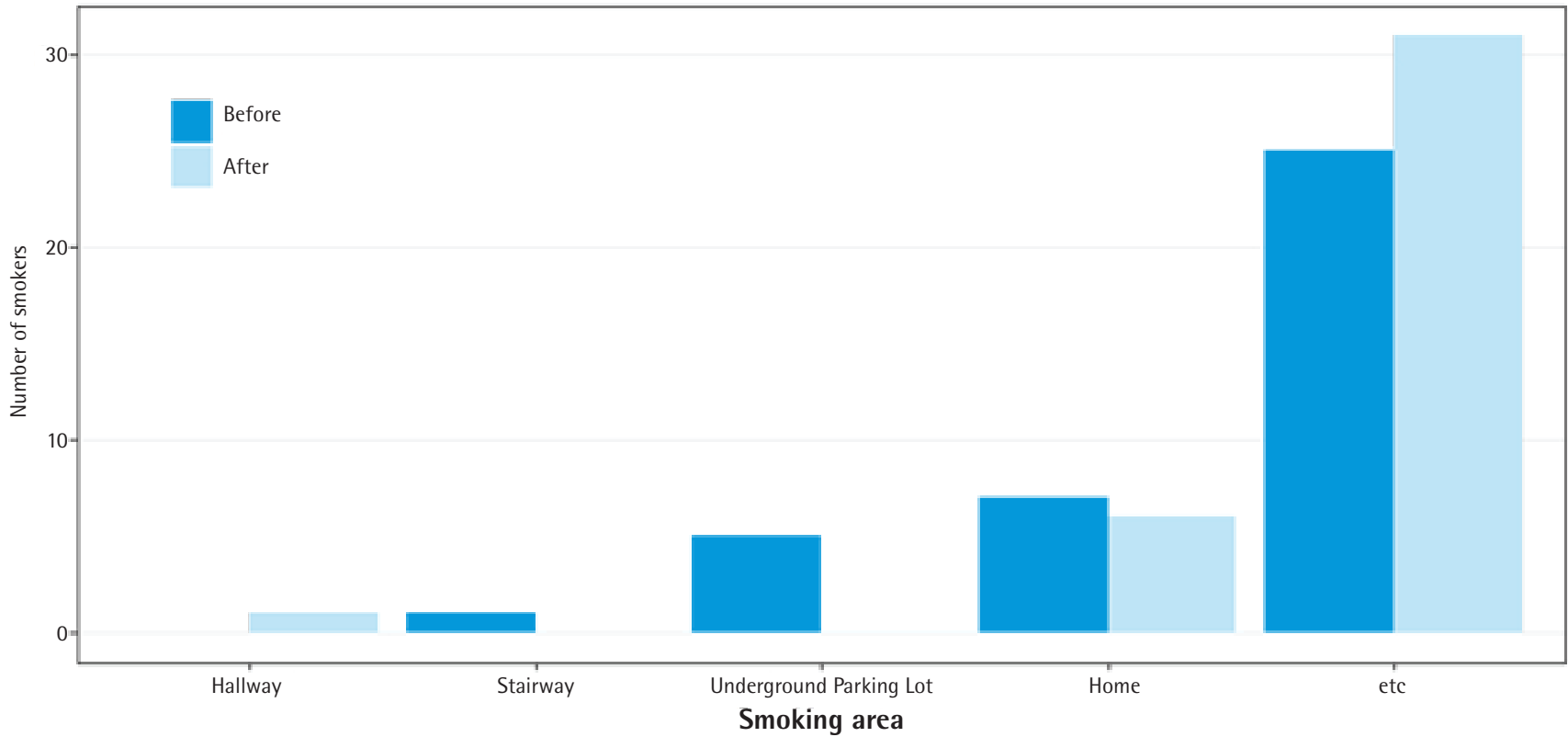

had attempted to quit after the designation of nonsmoking apartments. The remaining 23 (60.5\%) smokers indicated that there had been no change in the amount that they smoked after the designation of non-smoking apartments, but $9(23.7 \%)$ stated that the amount that they smoked had decreased (3 decreased their cigarette consumption in the home, and 6 their consumption outside). However, 6 smokers $(15.8 \%)$ reported increasing the amount smoked outside the home.

Before the designation of the non-smoking spaces, 6 individuals had smoked in public areas (hallway, $\mathrm{n}=0$; stairs, $n=1$; elevator, $n=5$ ). After the designation, this decreased to one person (hallway, $\mathrm{n}=1$; stairs, $\mathrm{n}=0$; elevator, $\mathrm{n}=0)$ (Figure 1) $(\mathrm{p}<0.001)$. The number of individuals who smoked in the home also decreased, from 7 to $6(\mathrm{p}<0.001)$. However, the number of people who smoked in places other than their homes and their apartment buildings' public areas increased from 28 to 32 following the designation of nonsmoking apartments $(\mathrm{p}<0.001)$. Places other than the public areas included the area outside the apartment complex and flower beds.

\section{DISCUSSION}

The results of this study suggest that after the designation of non-smoking areas in apartment buildings in Korea, smokers tended to refrain from smoking in public spaces designated as smoke-free areas, and instead smoked more in other places. The designation of non-smoking areas in apartment buildings generally had a positive effect on smoking habits, including smoking amount and smoking cessation attempts.

The prohibition of smoking in public areas, such as apartment buildings, renders smoking inconvenient and gradually changes smokers' habits ${ }^{4}$. In 2008, a mail survey was conducted, where 440 Oregon residents were invited to assess the effectiveness of the no-smoking policy introduced for apartment buildings in Oregon. The results showed that more than half of the smokers had decreased their smoking amount since the policy was implemented, while $14.7 \%$ of the smokers had succeeded in quitting ${ }^{5}$. Since the smoke-free housing policy was implemented in Waterloo, Canada, about a third of smokers therein reported a decrease in their cigarette consumption ${ }^{6}$. Smokers reported not only that their smoking had decreased as a result of the policy, but also that they had attempted to quit. Permanent non-smoking regulations in apartment buildings may encourage smokers to intensify their attempts to quit and thus influence the likelihood of smoking cessation.

The results of this study point to a need for smoking 
cessation support services for smokers as smoke-free spaces are expanded. Smokers perceive the necessity of smoking cessation, and thus attempt to quit, in response to the designation of non-smoking areas ${ }^{4,5}$, and the provision of support services may lead to a higher rate of success of smoking cessation attempts ${ }^{7}$. It is necessary to increase awareness, including smokers, regarding non-smoking regulations via public information campaigns. If designated nonsmoking areas are expanded, the government must promote any support services implemented to assist smokers with smoking cessation.

Smoking was strictly prohibited in the homes of most of the respondents in this study, thus compelling the smokers to smoke outside the home. Prohibition of smoking in public housing effectively prohibits smoking in places that are familiar and convenient for smokers, leading directly to a change in the locations in which they smoke; they are forced to find other suitable areas, such as hallways, stairs, or underground parking-lots. However, even before the designation of smoke-free spaces in apartment buildings, many smokers reported that they usually smoked in places other than the public areas. The designation of nonsmoking areas in public housing will be instrumental in inducing changes in the habits of regular smokers who live therein, but will not affect other smokers. These non-smoking areas are also likely to eliminate secondhand smoking hazards for non-smokers exposed to secondhand smoke in the public areas of their apartment buildings.

In the Oregon study, non-smokers' exposure to secondhand smoke was reduced from $41 \%$ to $17 \%$ after implementation of the anti-smoking policy ${ }^{5}$. Therefore, expansion of non-smoking areas in community housing will likely have a positive effect on non-smokers' exposure to secondhand smoke. In particular, if non-smoking regulations on households are reinforced and extended to public areas, the risk of exposure to secondhand smoke will be significantly decreased $^{8,9}$, and the associated socioeconomic costs will thus be mitigated ${ }^{9}$.

\section{Limitations}

This study had several limitations. First, the number of smokers who participated in the survey was small. Similar future studies should increase the number of subjects so that the results are more generalizable.
Second, owing to the limited number of regions surveyed, differences according to region were difficult to analyse. Third, the apartment buildings surveyed began enforcing the smoke-free policy on different dates thus, the extent of change in the smoking behavior of smokers may have differed depending on the difference in the duration of the buildings' designation as smoke-free. Also, this study's reliance on self-reported changes in smoking behavior limited the ability to make conclusions about changes before and after this designation. Moreover, we may have had a 'health participant' effect where those who had noticed changes to their exposure may have been more inclined to report and hence bias the results. Future research should use objective pre- and postsurveys to examine changes in the smoking behaviour of smokers after multi-unit buildings have been designated as smoke-free. In addition, this study was unable to determine the effect of smokers' smoking behavior on reducing the secondhand smoke exposure of non-smokers. Therefore, it will be necessary to use both objective and subjective measures to assess the secondhand smoke exposure of non-smokers before and after the designation of smoke-free areas in multiunit buildings to increase the impact of this policy.

\section{CONCLUSIONS}

The results of this study suggest that after the designation of non-smoking areas in apartment buildings in Korea, smokers tended to refrain from smoking in public spaces designated as smoke-free areas.

\section{REFERENCES}

1. WHO Framework Convention on Tobacco Control. https://www.who.int/fctc/text_download/en/. Published 2003. Updated 2005. Accessed November 14, 2019.

2. World Health Organization. 2018 Global progress report on implementation of the WHO Framework Convention on Tobacco Control. Geneva: World Health Organization; 2018. Licence: CG BY-NC-SA 3.0 IGO. https://www. who.int/fctc/reporting/WHO-FCTC-2018_global_ progress_report.pdf. Accessed November 14, 2019.

3. Snyder K, Vick JH, King BA. Smoke-free multiunit housing: a review of the scientific literature. Tob Control. 2016;25(1):920. doi:10.1136/tobaccocontrol-2014-051849

4. Kaufman P, Kang J, Kennedy RD, Beck P, Ferrence R. Impact of smoke-free housing policy lease exemptions on compliance, enforcement and smoking behavior: A qualitative study. Prev Med Rep. 2018;10:29-36. 
doi:10.1016/j.pmedr.2018.01.011

5. Pizacani BA, Maher JE, Rohde K, Drach L, Stark MJ. Implementation of a smoke-free policy in subsidized multiunit housing: effects on smoking cessation and secondhand smoke exposure. Nicotine Tob Res. 2012;14(9):1027-1034. doi:10.1093/ntr/ntr334

6. Kennedy RD, Ellens-Clark S, Nagge L, Douglas O, Madill C, Kaufman P. A Smoke-Free Community Housing Policy: Changes in Reported Smoking Behaviour-Findings from Waterloo Region, Canada. J Community Health. 2015;40(6):1207-1215. doi:10.1007/s10900-015-0050-0

7. Yerger VB, Battle RS, Moore RS. Evaluating the implementation process of a citywide smoke-free multiunit housing ordinance: insights from community stakeholders. Am J Public Health. 2014;104(10):18891891. doi:10.2105/ajph.2014.302075

8. Rosen LJ, Myers V, Winickoff JP, Kott J. Effectiveness of Interventions to Reduce Tobacco Smoke Pollution in Homes: A Systematic Review and Meta-Analysis. Int J Environ Res Public Health. 2015;12(12):16043-16059. doi:10.3390/ijerph121215038

9. King BA, Peck RM, Babb SD. National and state cost savings associated with prohibiting smoking in subsidized and public housing in the United States. Prev Chronic Dis. 2014;11. doi:10.5888/pcd11.140222
ACKNOWLEDGEMENTS

The authors thank the Korea Ministry of Health and Welfare and the Korea Health Promotion Institute.

\section{CONFLICTS OF INTEREST}

The authors have completed and submitted the ICMJE Form for Disclosure of Potential Conflicts of Interest and none was reported.

\section{FUNDING}

This study was supported by the Health Promotion Fund, Korea Health Promotion Institute, Republic of Korea.

AUTHORS' CONTRIBUTIONS

$\mathrm{JH}$ was responsible for the design of the study, data analysis, and interpretation of the results. A draft of the manuscript was written by $\mathrm{JH}$ and reviewed by EP. SC contributed to the interpretation of the results. All authors read and approved the final manuscript.

PROVENANCE AND PEER REVIEW

Not commissioned; externally peer reviewed. 